Journal of Research in Interprofessional

Practice and

Education

Vol. 3.1

March, 2013
Journal of Research in Interprofessional Practice and Education (JRIPE)

Vol. 3.1

(C) 2013

Corresponding author: Beth Davis. Email: ibmurray@@يncmaster.cai

\section{A Team Observed Structured Clinical Encounter (TOSCE) for Pre-Licensure Learners in Maternity Care: A Short Report on the Development of an Assessment Tool for Collaboration}

\author{
Beth Murray Davis, RM, PhD; Patricia Solomon, PhD; \\ Denise Marshall, MD; Anne Malott, RM, MScN; \\ Valerie Mueller, MD; Elizabeth Shaw, MD; \& Kelly Dore, PhD
}

\begin{abstract}
Background: Despite the support for Interprofessional Education (IPE) among policymakers, educators and professional regulating bodies, the research literature is limited with respect to the evaluation of effective assessment strategies. This short report outlines the development of a Team Observed Structured Clinical Encounter (TOSCE), which brings together learners from three health professions involved in primary care obstetrics - family physicians, midwives, and obstetricians-as a strategy for assessing collaborative competencies.

Methods: An interprofessional research team was brought together to develop and implement the TOSCE. The process by which the team generated TOSCE scenario stations is outlined, including the consensus-building process, based on a modified Delphi technique, to include expert input from others in the field of practice.

Findings: The scenarios developed by the research team for the TOSCE are highlighted including the assessment criteria, based on the Canadian Interprofessional Health Collaborative's National Competency Framework.

Conclusions: The TOSCE is an emerging and innovative learning tool that encourages the development of essential collaborative competencies. The process of developing a TOSCE outlined in this report offers an affordable, streamlined approach that could be used by educators in many disciplines as a summative or formative assessment strategy.
\end{abstract}

Keywords: Assessment of competencies; Maternity care; Teamwork; OSCE

\section{Introduction}

Interprofessional working occurs daily in the provision of maternity care. Expectant mothers and families in most parts of Canada can choose to have a midwife, a family physician, or an obstetrician provide primary maternity care during pregnancy and birth; however, collaborative care between these professions is essential. For example, family physicians and midwives have a scope of practice focused on low risk obstetrics, and both may need to work collaboratively with a consultant obstetrician when complications arise. Similarly, midwives may work collaboratively with family physicians when pre-existing medical conditions are present. Yet, the lack of teamwork and communication between professionals, as well as rigid adherence to professional hierarchies, and differing approaches to care have posed challenges within maternity services [1]. Several Canadian policy documents advocating for 
123

TOSCE for

Pre-Licensure

Learners in

Maternity Care

Murray Davis, Solomon, Marshall, Malott, Mueller, Shaw, \& Dore

Journal of Research in Interprofessional Practice and Education

Vol. 3.1

March, 2013 an interprofessional workforce specifically addressed the need for interprofessional training in maternity care [2], and recommended creating training opportunities for collaborative practice for both learners and professionals. These documents emphasized the value of a curriculum that develops skills for working collaboratively and an understanding of the scope of practice and philosophy of care of all maternity care providers [2]. Despite the evident support for Interprofessional Education (IPE) among policymakers, educators, and professional regulating bodies, there remains a dearth of effective assessment strategies used to determine if IPE activities are effective in promoting collaboration among learners.

The Objective Structured Clinical Examination or OSCE has been widely used as a valid and reliable tool within health profession education for the assessment of knowledge, skills, and attitudes through structured observation. Traditionally, an OSCE involves an individual student moving through a series of scenarios, while being observed by assessors [3]. The idea of a group OSCE has been used previously, and the "interprofessional" team OSCE has had some use to date, with interest growing [3-6]. The goal of the interprofessional approach is to bring together individuals from different professions and to encourage participants to work together as a team. The competencies assessed are focused on teamwork and collaboration, rather than discrete clinical skills.

The current project builds on this previous work, specifically that done by the McMaster-Ottawa research team that developed the Team Observed Structured Clinical Encounter (TOSCE), which has been used for both undergraduate students in Palliative Care and for Primary Health Care teams since 2006 [5,7,8]. One of the key features of the McMaster-Ottawa TOSCE is the use of a standardized observer score sheet, which contains a checklist in a table format of collaborative competencies, as well as a global rating scale for the performance of each student and the team overall [8]. The competencies assessed on the score sheet are based on the Canadian Interprofessional Health Collaborative (CIHC) National Collaborative Competencies, which include: communication, collaboration, roles/responsibilities, collaborative patient-family centred approach, conflict management/resolution, and team function [9].

The McMaster-Ottawa TOSCE has a high degree of acceptability and feasibility, based on evaluations from learners and assessors [7]. In particular, students have reported that the feedback received during the TOSCE was among the most valuable learning in their training [7]. Further, psychometric testing of the McMasterOttawa TOSCE has established the validity and reliability of the standardized score sheet checklist and evidence of inter-rater reliability and internal consistency [5].

The aim of this article is to report on the development of a TOSCE for learners from three health professions-family physicians, midwives, and obstetricians-at an Ontario University who are involved in primary care obstetrics. The methods and results described below outline the planning and development process of the project. The maternity care TOSCE development process described here is targeted for pre-licensure learners, and offers an affordable and streamlined process for educators. 
124

TOSCE for

Pre-Licensure

Learners in

Maternity Care

Murray Davis, Solomon, Marshall, Malott, Mueller, Shaw, \& Dore
Journal of Research in Interprofessional Practice and Education

Vol. 3.1

March, 2013

\section{Methods}

A consensus process, based on a modified Delphi technique, was chosen as the best design to develop the TOSCE station scenario content, given that the modified Delphi has been used successfully by other groups during the development of team OSCE stations [4-5]. The strengths of this process include gaining input from experts, while facilitating consensus decision-making through modification and group process [10-11].

Our process began by bringing together an interprofessional group of educators as the core research team to plan the pilot initiative. The team consisted of a convenience sample of content experts from the three professional groups, and IPE and TOSCE experts. They began by identifying brief topic areas for possible TOSCE stations, based on their clinical expertise. The goal was to generate topic areas that could be developed into stations that represent commonly encountered clinical issues and require interprofessional collaboration during the management of patient care. Next, the topic list was distributed via email to a convenience sample of experts in the field of maternity education and clinical practice for comment and review. The experts were asked open-ended questions about the topic areas, with specific attention to the clinical relevance and to the possibility that the clinical management would result in collaborative practice. They provided comments on each topic and suggestions for additional topics. Following this feedback, the research team held a meeting to develop the topic areas into the scenario content for the TOSCE stations. After development of the final scenarios by the research team, the scenarios were again circulated to the experts in the field for comment and review, based on open-ended questions pertaining to the value of the scenario as a learning tool for collaboration and the relevance of the clinical case. The core team reviewed these comments, and modifications to the scenarios were made.

\section{Results}

\section{Developing the TOSCE}

The core research team $(n=7)$ developed a list of 13 possible topic areas. The topics included issues such as pre-existing medical conditions, obstetric emergencies, complex social issues, postpartum depression, and complicated fetal or newborn conditions. The topic list was then distributed to the experts in the field for review. The group of experts included seven midwives, ten family physicians who provide primary obstetric care, and four obstetricians. Based on the feedback of the experts in the field, five additional topics were added.

The core team met again to review the topic list with the intention of turning the topics into fully developed scenarios that would make up the content of the TOSCE stations. The eight-hour "immersion" meeting, which took place in an offsite location, was beneficial for minimizing daily distractions and scheduling difficulties that result when attempting to hold several short meetings over a series of weeks. It also allowed the team to spend a substantial amount of time focused on the project. 
JRIPE

125

TOSCE for

Pre-Licensure

Learners in

Maternity Care

Murray Davis, Solomon, Marshall, Malott, Mueller, Shaw, \& Dore

From the 18 topics originally generated, seven scenarios were developed. The other 11 topics were rejected for two main reasons. First, some of them only presented an opportunity for collaboration between two professional groups, such as the management of hypothyroidism where a family physician and midwife would work together, but there would be a limited role for an obstetrician. Second, others did not lend themselves to collaborative management of care. For example, the management of complex cardiac issues typically requires the transfer of care to a highrisk obstetrician, with little ongoing input from either the family physician or midwife.

The seven scenarios were sent by email to the same group of experts who provided comments and minor editing changes. Based on these comments, there was consensus among the core team and the experts that the scenarios were realistic, could be managed with collaboration, and did not contain errors or omissions.

\section{The scenarios}

The scenarios developed for the TOSCE are presented in Table 1.

\section{Table 1: Summary of TOSCE stations including topic and collaborative competencies assessed}

\begin{tabular}{|c|l|l|}
\hline Station & Topic & Collaborative Competency Assessed \\
\hline Nadja & $\begin{array}{l}\text { Recent immigrant, requesting } \\
\text { culturally sensitive care }\end{array}$ & $\begin{array}{l}\text { Communication, recognition of roles of others, patient- } \\
\text { centred approach, conflict management, team functioning. }\end{array}$ \\
\hline Katrina & Substance use & $\begin{array}{l}\text { Communication, collaboration, conflict management, } \\
\text { team functioning. }\end{array}$ \\
\hline Darla & Fetal demise & $\begin{array}{l}\text { Communication, collaboration, understanding of roles } \\
\text { and responsibilities, patient-centred approach. }\end{array}$ \\
\hline Jessica & $\begin{array}{l}\text { Hypertension, discharge } \\
\text { planning }\end{array}$ & $\begin{array}{l}\text { Communication, collaboration, understanding of roles and } \\
\text { responsibilities, patient-centred approach, team functioning. }\end{array}$ \\
\hline Maya & $\begin{array}{l}\text { Complex mental health issues, } \\
\text { refusing care }\end{array}$ & $\begin{array}{l}\text { Communication, collaboration, understanding of roles } \\
\text { and responsibilities, patient-centred approach. }\end{array}$ \\
\hline Olivia & Postpartum depression & $\begin{array}{l}\text { Communication, collaboration, understanding of roles } \\
\text { and responsibilities, patient-centred approach. }\end{array}$ \\
\hline Crystal & Obesity & $\begin{array}{l}\text { Communication, understanding of roles and } \\
\text { responsibilities, patient-centred approach. }\end{array}$ \\
\hline
\end{tabular}

The scenarios were written to be 20 minutes in duration, followed by 10 minutes

Journal of Research in Interprofessional Practice and Education

Vol. 3.1

March, 2013 of feedback. They were also written to include a role for three team members, one from each profession, who would work through the scenario together. All scenarios included a written description of instructions for participants. The stimulus format for how the patient case was presented in the scenarios varied; some used a written description of a patient case, others a standardized patient, and one a video mono- 
TOSCE for

Pre-Licensure

Learners in

Maternity Care

Murray Davis, Solomon, Marshall, Malott, Mueller, Shaw, \& Dore
Journal of Research in Interprofessional Practice and Education

Vol. 3.1

March, 2013

\section{Journal of Research in Interprofessional Practice and Education}

logue from a patient.

Scenarios were not intended to assess clinical knowledge or management, but rather to evaluate the collaborative competencies through the use of the McMasterOttawa observer score sheet (Table 2) [5]. The intent was that two evaluators, also drawn from the three professional groups, would observe each station, provide feedback, and complete the score sheet.

\section{Table 2: Sample scenario with written description and instructions for learners}

\section{Jessica}

You are seeing Jessica on the postpartum ward. She delivered a healthy 8-5 baby boy at term by vacuum assisted delivery under obstetrical care. She has a long-standing history of hypertension, well controlled under the management of her family physician. She chose midwifery care for this pregnancy. A transfer of care to obstetrics occurred at 35 weeks due to escalating hypertension, requiring medication with Labetalol. Her midwife remains involved for supportive care. Her blood pressure has remained stable postpartum with medication. She is requesting discharge home today. The nurse approaches the three of you - her family physician, her midwife, and her obstetrician - in the nurse's station and asks "so what's the plan for Jessica's follow-up and hypertension management after discharge?"

Please discuss the management strategy for Jessica.

\section{Discussion}

This paper presents the development of an emerging and innovative learning tool that promotes essential collaborative competencies. The TOSCE goes beyond the traditional IPE assessment of attitudes and stereotypes and instead provides a way of assessing changes in knowledge and behaviour. This TOSCE project offers an affordable, streamlined approach that could be used by educators in many disciplines.

As with other IPE projects, there were challenges that impacted the development and implementation of the TOSCE. Specifically, keeping the scenarios realistic and clinically relevant was occasionally problematic as the realities of clinical practice meant that the three professionals involved were not often in the same room at the same time. Two key features of the development process provided valuable lessons for planning future events such as this. First, the use of the consensus process to develop stations was useful due to its minimal cost and the minimal time commitment required from the experts. As a result, we had an $80 \%$ response rate from the experts surveyed. In addition, the anonymity of responses, collected electronically, may have minimized the impact of power struggles or hierarchies between the three professional groups of experts [10-11]. Participants were able to be honest without fear of how colleagues would view their responses. This was a useful lesson for interprofessional planning teams. The second lesson learned pertains to the value of the immersion meeting where the stations were developed. This was a highly productive scheduling strategy that resulted in synergy between members of 
127

TOSCE for

Pre-Licensure

Learners in

Maternity Care

Murray Davis, Solomon, Marshall, Malott, Mueller, Shaw, \& Dore
Journal of Research in Interprofessional Practice and Education

Vol. 3.1

March, 2013 the team, which was important in developing creative and effective scenarios. At the end of the eight-hour meeting, the stations had gone from a simple topic to a fully written scenario, with descriptions for participants and observers, and with suitable stimulus for presenting the case, such as a script for a video or a standardized patient. These two strategies improved the feasibility of the planning process and improved the TOSCE stations.

\section{Next steps}

The next steps for our project include an assessor training day where faculty in the three professional groups will be introduced to the rationale and evidence to support the project and will participate in a mock TOSCE. The pilot project will then be implemented with learners. The TOSCE will be run twice, first as a formative experience using three stations, and then as a summative encounter using the remaining four stations. The TOSCE will be a voluntary event for a small group of 18 learners who will rotate through stations in small groups with one participant from each profession. The same learners will participate in the second TOSCE several weeks later. The repetition of the stations during the two events will facilitate the evaluation of inter-rater reliability. Pre- and post-questionnaires will also be completed by learners and faculty to evaluate acceptability, feasibility, and educational impact of the project. Following a favourable evaluation, the TOSCE will be run again in future years as a mandatory IPE event for midwifery students and family medicine and obstetric residents.

\section{Acknowledgement}

The authors wish to acknowledge the funding support for this research, which was granted by the Program for Interprofessional Education and Research (PIPER) at McMaster University.

\section{References}

1. O’Neill, O. [Editor]. (2008). Safe Maternity Teams. Safe Births: Everybody's Business. An independent inquiry into the safety of maternity services in England (chapter 3, pp. 27-39). London: Kings Fund.

2. Multidisciplinary Collaborative Primary Maternity Care Project. (2006). Executive Report. URL: www.mcp2.ca (March 19, 2008).

3. Symonds, I., Cullen, L., \& Fraser, D. (2003). Evaluation of a formative interprofessional team objective structured clinical examination (ITOSCE): A method of shared learning in maternity education. Med Teach, 25(1), 38-41.

4. Simmons, B., Egan-Lee, E., Wagner, S., Esdaile, M., Baker, L., \& Reeves, S. (2010). Assessment of interprofessional learning: The design of an interprofessional objective structured clinical examination (iOSCE) approach. Journal of Interprofessional Care, July 20, 1-2.

5. Solomon, P., Marshall, D., Boyle, A., Burns, S., Casimiro, L., Hall, P., \& Weaver, L. (2011). Establishing Face and Content Validity of the McMaster-Ottawa Team Observed Clinical Encounter (TOSCE). Journal of Interprofessional Care, July 25(4), 302-304.

6. Singleton, A., Smith, F., Harris, T., Ross-Harper, R., \& Hilton, S. (1999). An evaluation of the Team Objective Structured Clinical Examination (TOSCE). Medical Education, 33, 34-41.

7. Marshall, D., Hall, P., \& Taniguchi, A. (2008). Team OSCEs: Evaluation methodology or educational encounter? Medical Education, 42(11), 1129-1130. 
128

TOSCE for Pre-Licensure Learners in Maternity Care

Murray Davis, Solomon, Marshall, Malott, Mueller, Shaw, \& Dore
8. Hall, P., Marshall, D., Weaver, L., Boyle, A., \& Taniguchi, A. (2011). A method to enhance student teams in Palliative Care: Piloting the McMaster-Ottawa team observed structured clinical encounter. Journal of Palliative Care, 14(6), 744-750.

9. Canadian Interprofessional Health Collaborative. (2010). A national interprofessional competency framework. Vancouver, BC: College of Health Disciplines, University of British Columbia.

10. Williams, P.L., \& Webb, C. (1994). The Delphi technique: A methodological discussion. Journal of Advanced Nursing, 19(1), 180-186.

11. Beretta, R. (1996). A critical review of the Delphi technique. Nurse Researcher, 3(4), 79-89. 